Bull. Korean Math. Soc. 49 (2012), No. 4, pp. 737-748

http://dx.doi.org/10.4134/BKMS.2012.49.4.737

\title{
EXISTENCE OF WEAK NON-NEGATIVE SOLUTIONS FOR A CLASS OF NONUNIFORMLY BOUNDARY VALUE PROBLEM
}

\author{
Trinh Thi Minh Hang and Hoang Quoc Toan
}

\begin{abstract}
The goal of this paper is to study the existence of non-trivial non-negative weak solution for the nonlinear elliptic equation:

$$
-\operatorname{div}(h(x) \nabla u)=f(x, u) \text { in } \Omega
$$

with Dirichlet boundary condition in a bounded domain $\Omega \subset \mathbb{R}^{N}, N \geq 3$, where $h(x) \in L_{l o c}^{1}(\Omega), f(x, s)$ has asymptotically linear behavior. The solutions will be obtained in a subspace of the space $H_{0}^{1}(\Omega)$ and the proofs rely essentially on a variation of the mountain pass theorem in [12].
\end{abstract}

\section{Introduction}

Let $\Omega$ be a bounded domain in $\mathbb{R}^{N}, N \geq 3$ with smooth boundary $\partial \Omega$. We study the existence of non-trivial weak solution of the following Dirichlet problem

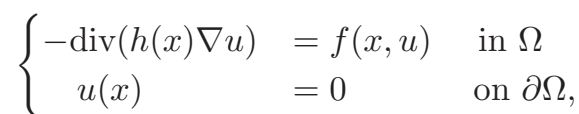

where $h(x) \in L_{l o c}^{1}(\Omega), h(x) \geq 1$ a.e. $x \in \Omega$.

Due to the presence of $h(x) \in L_{l o c}^{1}(\Omega)$, the problem now may be non-uniform in sense that the functional associated to the problem may be infinity for some $u$ in $H_{0}^{1}(\Omega)$. In what follow, we deduce the problem (1.1) to a uniform one by using an appropriate weighted Sobolev space. Then applying a variation of the mountain pass theorem in [12], we prove that the problem (1.1) admits a non-trivial non-negative weak solution in a subspace of the $H_{0}^{1}(\Omega)$. Let us introduce some hypotheses:

Received March 26, 2011; Revised January 5, 2012.

2010 Mathematics Subject Classification. 35J20, 35J65.

Key words and phrases. mountain pass theorem, the weakly continuously differentiable functional.

Research supported by the National Foundation for Science and Technology Development of Vietnam (NAFOSTED). 
F1) $f: \Omega \times \mathbb{R} \longrightarrow \mathbb{R}$ is a Caratheodory function satisfying $f(x, s)=0$ for all $s \leq 0$, a.e. $x \in \Omega$.

F2) There exists a constant $C>0$ such that $\left|\frac{f(x, s)}{s}\right| \leq C$ a.e. $x \in \Omega$, $\forall s \in(0,+\infty)$ and $f$ is "asymptotically linear" in the sense that there exists $\beta \in C(\bar{\Omega})$ such that $\beta(x)=\lim _{s \rightarrow+\infty} \frac{f(x, s)}{s}$ uniformly a.e. $x \in \Omega$.

Firstly, we introduce some following remark:

Remark 1.1. There is a rich literature dealing with asymptotically linear problem and existence results on bounded domain or unbounded domain in the case that $h(x)=1$ which have been obtained via variational methods (see $[1,4,18,19,20]$ and the reference therein). However, to the best of our knowledge, there has never been any study on the existence results of asymptotically linear of the problem (1.1) in the case $h(x) \in L_{l o c}^{1}(\Omega)$. This case will be appropriate in our paper.

Remark 1.2. The problem (1.1) when the nonlinearity satisfies the condition

$$
0<\mu F(x, s) \leq f(x, s) s \quad \text { for } \mu>2,|s| \geq M,
$$

where $F(x, s)=\int_{0}^{s} f(x, t) d t$ has been studied either when $h(x) \in L^{\infty}(\Omega)$ or $h(x) \in L_{l o c}^{1}(\Omega)$ (see $\left.[9,21,22]\right)$. We point out that the condition (1.2) implies that $f$ has to be superlinear at infinity. So this kind of assumption is not appropriate in our situation.

Let $H_{0}^{1}(\Omega)$ be the usual Sobolev space under the norm

$$
\|u\|=\left(\int_{\Omega}\left(|\nabla u|^{2}+|u|^{2}\right) d x\right)^{\frac{1}{2}} .
$$

We now consider following subspaces $H$ of $H_{0}^{1}(\Omega)$

$$
H=\left\{u \in H_{0}^{1}(\Omega): \int_{\Omega} h(x)|\nabla u|^{2} d x<+\infty\right\} .
$$

Then $H$ is a Hilbert space with the norm

$$
\|u\|_{H}^{2}=\int_{\Omega} h(x)|\nabla u|^{2} d x
$$

and the scalar product (see $[9,22])$

$$
\langle u, v\rangle_{H}=\int_{\Omega} h(x) \nabla u \nabla v d x, \quad u, v \in H .
$$

Furthermore we have $\|u\|_{H_{0}^{1}(\Omega)} \leq\|u\|_{H}, u \in H$ and the continuous embedding $H \hookrightarrow H_{0}^{1}(\Omega) \hookrightarrow L^{q}(\Omega), 2 \leq q \leq 2^{*}=\frac{2 N}{N-2}$ hold true. Moreover, the embedding $H \hookrightarrow L^{2}(\Omega)$ is compact.

Definition 1.1. We say that $u \in H$ is a weak solution of the problem (1.1) if

$$
\int_{\Omega} h(x) \nabla u \nabla \varphi d x-\int_{\Omega} f(x, u) \varphi d x=0
$$


for all $\varphi \in H$.

\section{Auxiliary results}

We define the functional $J: H \longrightarrow \mathbb{R}$ given by

$$
\begin{aligned}
J(u) & =\frac{1}{2} \int_{\Omega} h(x)|\nabla u|^{2} d x-\int_{\Omega} F(x, u) d x \\
& =T(u)-P(u), \quad u \in H
\end{aligned}
$$

where

$$
\begin{aligned}
F(x, t) & =\int_{0}^{t} f(x, s) d s, \quad T(u)=\frac{1}{2} \int_{\Omega} h(x)|\nabla u|^{2} d x, \\
P(u) & =\int_{\Omega} F(x, u) d x, \quad u \in H .
\end{aligned}
$$

Firstly we remark that the critical points of the functional $J$ correspond to the weak solution of the problem (1.1). Moreover, due to the presence of $h(x) \in L_{l o c}^{1}(\Omega)$, in general, the functional $T$ (and thus $J$ ) does not belong to $C^{1}(H)$. This means that we cannot apply the classical mountain pass theorem by Ambrossetti-Rabinowitz. In order to overcome this difficulty, we shall apply a weak version of the mountain pass theorem introduced by D. M. Duc [12]. But we first recall the following useful concept of weak continuous differentiability:

Definition 2.1. Let $J$ be a functional from a Banach space $Y$ into $\mathbb{R}$. We say that $J$ is weakly continuously differentiable on $Y$ if and only if three following conditions are satisfied:

i) $J$ is continuous on $Y$.

ii) For any $u \in Y$ there exists a linear map $D J(u)$ from $Y$ into $\mathbb{R}$ such that

$$
\lim _{t \rightarrow 0} \frac{J(u+t \varphi)-J(u)}{t}=\langle D J(u), \varphi\rangle, \forall \varphi \in Y .
$$

iii) For any $\varphi \in Y$, the map $u \mapsto\langle D J(u), \varphi\rangle$ is continuous on $Y$.

We denote by $C_{w}^{1}(Y)$ the set of weakly continuously differentiable functionals on $Y$. It is clear that $C^{1}(Y) \subset C_{w}^{1}(Y)$, where $C^{1}(Y)$ is the set of all continuously Fréchet differentiable functionals on $Y$. With similar arguments as those used in the proof of Proposition 2.2 in [22], we conclude the following proposition which concerns the smoothness of the functional $J$.

Proposition 2.1. The functional J given by (2.4) is weakly continuously differentiable on $H$ and we have

$$
\langle D J(u), \varphi\rangle=\int_{\Omega} h(x) \nabla u \nabla \varphi d x-\int_{\Omega} f(x, u) \varphi d x \quad \text { for all } u, \varphi \in H .
$$

By Proposition 2.1, the critical points of the functional $J$ correspond to the weak solutions of the problem (1.1). 
Proposition 2.2 (see Lemma 2.3 in [9]). The functional $T$ given by (2.5) is weakly lower semicontinuous on the space $H$.

Proposition 2.3. Let $v \in L^{\infty}(\Omega)$ such that $\Omega^{+}=\{x \in \Omega: v(x)>0\}$ is an open set in $\mathbb{R}^{N}$. Set

$$
\Lambda:=\inf _{u \in H}\left\{\int_{\Omega} h(x)|\nabla u|^{2} d x: \int_{\Omega} v(x) u^{2} d x=1\right\} .
$$

Then

i) $S=\left\{u \in H: \int_{\Omega} v(x) u^{2} d x=1\right\} \neq \emptyset$,

ii) there exists $u_{0} \in S: \int_{\Omega} h(x)\left|\nabla u_{0}\right|^{2} d x=\Lambda$ and $u_{0} \geq 0, u_{0} \neq 0$ in $\Omega$.

Proof. i) Let $u \in C_{0}^{\infty}\left(\Omega^{+}\right), u \neq 0$ and $u \in H$, then $\int_{\Omega^{+}} v(x) u^{2} d x>0$.

Then

Choose $\bar{u} \in H$ as $\bar{u}=\frac{u(x)}{\left(\int_{\Omega^{+}} v(x) u^{2} d x\right)^{\frac{1}{2}}}$ as $x \in \Omega^{+}$and $\bar{u}=0$ as $x \in \Omega \backslash \Omega^{+}$.

$$
\int_{\Omega} v(x) \bar{u}^{2} d x=\int_{\Omega^{+}} v(x) \frac{u^{2}}{\int_{\Omega^{+}} v(x) u^{2} d x} d x=1 .
$$

Hence $S \neq \emptyset$.

ii) Let $\left\{u_{m}\right\} \subset H$ be a minimizing sequence, i.e.,

$$
\int_{\Omega} h(x)\left|\nabla u_{m}\right|^{2} d x \longrightarrow \Lambda \text { and } \int_{\Omega} v(x) u_{m}^{2} d x=1 .
$$

So $\left\{u_{m}\right\}$ is bounded in $H$. Then there exists a subsequence of $\left\{u_{m}\right\}$ still denoted by $\left\{u_{m}\right\}$ such that $u_{m} \rightarrow \hat{\mathrm{u}}$ in $H$ and $u_{m} \rightarrow \hat{\mathrm{u}}$ in $L^{2}(\Omega)$. We have $\hat{u} \in S$. Indeed,

$$
1=\lim _{m \rightarrow+\infty} \int_{\Omega} v(x) u_{m}^{2} d x=\int_{\Omega} v(x) \hat{\mathrm{u}}^{2} d x
$$

(from $v \in L^{\infty}(\Omega)$ we deduce $\int_{\Omega} v(x)\left(u_{m}^{2}-\hat{\mathrm{u}}^{2}\right) d x \rightarrow 0$ ). Then by the minimizing properties of $\left\{u_{m}\right\}$ and by the weakly lower semicontinuity of the functional $\int_{\Omega} h(x)|\nabla u|^{2} d x$ (see Proposition 2.2) we have

$$
\Lambda=\lim \inf _{m \rightarrow+\infty} \int_{\Omega} h(x)\left|\nabla u_{m}\right|^{2} d x \geq \int_{\Omega} h(x)|\nabla \hat{\mathrm{u}}|^{2} d x \geq \Lambda .
$$

So we get $\Lambda=\int_{\Omega} h(x)|\nabla \hat{\mathrm{u}}|^{2} d x$.

We have $\hat{\mathrm{u}} \in H$ and $\hat{\mathrm{u}}$ is a minimizer of

$$
\inf \left\{\int_{\Omega} h(x)|\nabla u|^{2} d x: \int_{\Omega} v(x) u^{2} d x=1\right\} .
$$

We show that $|\hat{\mathrm{u}}|$ is a minimizer too. Since $\hat{\mathrm{u}} \in H \subset H_{0}^{1}(\Omega)$ then $|\hat{\mathrm{u}}| \in H_{0}^{1}(\Omega)$ (see Lemma 7.6, p. 145 in [14]).

Moreover

$$
\int_{\Omega} v(x) \hat{\mathrm{u}}^{2} d x=\int_{\Omega} v(x)|\hat{\mathrm{u}}|^{2} d x, \text { so }|\hat{\mathrm{u}}| \in S .
$$


Finally

$$
\Lambda=\int_{\Omega} h(x)|\nabla \hat{\mathrm{u}}|^{2}=\left.\int_{\Omega} h(x)|\nabla| \hat{\mathrm{u}}\right|^{2} d x .
$$

So $|\hat{\mathrm{u}}| \in H$ and $|\hat{\mathrm{u}}|$ is a minimizer then $\hat{\mathrm{u}} \geq 0$. We suppose that $\hat{\mathrm{u}}=0$ then we deduce that $\int_{\Omega} v(x) \hat{\mathrm{u}}^{2} d x=0$, a contradiction. Set $u_{0}=|\hat{\mathrm{u}}|, u_{0} \geq 0$ and $u_{0} \neq 0$ in $\Omega$. The proof of Proposition 2.3 is complete.

\section{Main results}

Let us introduce following hypotheses.

F3) There exists $x_{0} \in \Omega$ such that $\beta\left(x_{0}\right)>0$ where $\beta$ is defined by F2). Denoted by

$$
\Omega_{\beta}=\{x \in \Omega: \beta(x)>0\}
$$

and assume that

$$
\Lambda_{\beta}=\inf _{u \in H\left(\Omega_{\beta}\right)} \frac{\int_{\Omega_{\beta}} h(x)|\nabla u|^{2} d x}{\int_{\Omega_{\beta}} \beta(x) u^{2} d x}>1 .
$$

F4) There exist two positive constants $\tau_{1}, \tau_{2}$ such that

$$
\begin{aligned}
\lim _{s \rightarrow 0} \frac{2 F(x, s)}{s^{2}} & \leq \tau_{1}<\lambda_{1}<\tau_{2} \leq \lim _{s \rightarrow+\infty} \frac{2 F(x, s)}{s^{2}} \text { uniformly a.e. } x \in \Omega, \\
\text { where } \lambda_{1} & =\inf _{u \in H} \frac{\int_{\Omega} h(x)|\nabla u|^{2} d x}{\int_{\Omega} u^{2} d x} .
\end{aligned}
$$

Our main result is given by the following theorem.

Theorem 3.1. Assuming hypotheses F1)-F4) are fulfilled. Then the problem (1.1) has at least one non-negative non-trivial weak solution in space $H$.

In order to prove Theorem 3.1, we need some following propositions.

Proposition 3.1. Assuming F1), F2), F4) are fulfilled. Then there exist $\alpha, \rho>$ 0 such that $J(u) \geq \alpha$ if $\|u\|_{H}=\rho$. Moreover, there exists $\varphi_{0} \in H$ such that $J\left(t \varphi_{0}\right) \rightarrow-\infty$ as $t \rightarrow+\infty$.

Proof. By F4) $\lim _{s \rightarrow+\infty} \frac{2 F(x, s)}{s^{2}} \geq \tau_{2}$ uniformly a.e. $x \in \Omega$, we deduce that there exists $s_{0}>0$ such that $\frac{2 F(x, s)}{s^{2}} \geq \tau_{2}$ for all $s>s_{0}$ or $F(x, s) \geq \frac{1}{2} \tau_{2} s^{2}$ for all $s>s_{0}$ uniformly a.e. $x \in \Omega$. We choose $t_{0} \in\left(0, s_{0}\right]$ such that $F\left(x, t_{0}\right)<\frac{1}{2} \tau_{2} t_{0}^{2}$ a.e. $x \in \Omega$.

Fix $\varepsilon>0$. There exists $B\left(\varepsilon, t_{0}\right)$ such that $F\left(x, t_{0}\right) \geq \frac{1}{2}\left(\tau_{2}-\varepsilon\right) t_{0}^{2}-B\left(\varepsilon, t_{0}\right)$. Denote $B(\varepsilon)=\sup _{t_{0} \leq s_{0}} B\left(\varepsilon, t_{0}\right)$. We obtain for any given $\varepsilon>0$ there exists $B=B(\varepsilon)$ such that

$$
F(x, s) \geq \frac{1}{2}\left(\tau_{2}-\varepsilon\right) s^{2}-B \text { for all } s \in(0,+\infty) \text { a.e. } x \in \Omega .
$$


Remark that by F2) we deduce $\lim _{s \rightarrow+\infty} \frac{F(x, s)}{s^{q}}=0$ a.e. $x \in \Omega$ and $q>2$. Fix arbitrarily $\varepsilon>0$. In the same way, using the second inequality of F4) and F2), It follows that there exists $A=A(\varepsilon)>0$ such that

$$
2 F(x, s) \leq\left(\tau_{1}+\varepsilon\right) s^{2}+2 A(\varepsilon) s^{q} \text { for all } s>0 \text { a.e. } x \in \Omega .
$$

For any given $\varepsilon>0$ there exists $A=A(\varepsilon)>0, B=B(\varepsilon)>0$ such that $\frac{1}{2}\left(\tau_{2}-\varepsilon\right) s^{2}-B \leq F(x, s) \leq \frac{1}{2}\left(\tau_{1}+\varepsilon\right) s^{2}+A s^{q}$ for all $s \in(0,+\infty)$ a.e. $x \in \Omega$. Now we choose $\varepsilon>0$ so that $\tau_{1}+\varepsilon<\lambda_{1}<\tau_{2}-\varepsilon$, we have

$$
\begin{aligned}
J(u) & =\frac{1}{2}\|u\|_{H}^{2}-\int_{\Omega} F(x, u) d x \\
& \geq \frac{1}{2}\|u\|_{H}^{2}-\frac{1}{2} \int_{\Omega}\left(\varepsilon+\tau_{1}\right) u^{2} d x-\int_{\Omega} A|u|^{q} d x \\
& \geq \frac{1}{2}\left(1-\frac{\varepsilon+\tau_{1}}{\lambda_{1}}\right)\|u\|_{H}^{2}-A k\|u\|_{H}^{q} .
\end{aligned}
$$

With $q>2$, choose $\rho=\|u\|_{H}$ small enough then we have

$$
\alpha=\frac{1}{2}\left(1-\frac{\varepsilon+\tau_{1}}{\lambda_{1}}\right)\|u\|_{H}^{2}-A k\|u\|_{H}^{q}>0 .
$$

Moreover,

$$
J(u) \leq \frac{1}{2}\|u\|_{H}^{2}-\frac{1}{2} \int_{\Omega}\left(\tau_{2}-\varepsilon\right)|u|^{2} d x+B|\Omega| .
$$

Choose $\varphi_{0} \in C_{0}^{\infty}(\Omega), \varphi_{0}>0$ such that $\varphi_{0}$ is a $\lambda_{1}$-eigen-function, that is, it satisfies $\lambda_{1} \int_{\Omega} \varphi_{0}^{2} d x=\int_{\Omega} h(x)|\nabla u|^{2} d x$. Denote $u_{0}=t \varphi_{0}$ then

$$
J\left(u_{0}\right) \leq \frac{1}{2}\left(1-\frac{\tau_{2}-\varepsilon}{\lambda_{1}}\right)|t|^{2}\left\|\varphi_{0}\right\|^{2}+B|\Omega| \rightarrow-\infty \text { as } t \rightarrow+\infty .
$$

Proposition 3.2. Assuming hypotheses F1)-F4) are fulfilled. Let $\left\{u_{m}\right\}$ be a Palais Smale sequence in $H$, i.e.,

$$
\lim _{m \rightarrow \infty} J\left(u_{m}\right)=c, \quad \lim _{m \rightarrow+\infty}\left\|D J\left(u_{m}\right)\right\|_{H^{*}}=0 .
$$

Suppose that $\left\{u_{m}\right\}$ is not bounded in $H$. Then there exists a subsequence of $\left\{u_{m}\right\}$ until denoted $\left\{u_{m}\right\}$ such that $\left\|u_{m}\right\|_{H} \rightarrow+\infty$ as $m \rightarrow+\infty$. Putting $w_{m}=\frac{u_{m}}{\left\|u_{m}\right\|_{H}}$. Then there exists a subsequence $\left\{w_{m_{k}}\right\}$ of $\left\{w_{m}\right\}$ such that $\left\{w_{m_{k}}\right\} \rightarrow w$ in $H$ satisfying

i) $w \neq 0$ in $\Omega$,

ii) $w>0$ in $\Omega$,

iii) $-\operatorname{div}(h(x) \nabla w)=\beta(x) w$ in $\Omega$.

Proof. We have $\left\|w_{m}\right\|_{H}=1$, so $\left\{w_{m}\right\}$ is bounded in $H$ then there exists a subsequence $\left\{u_{m_{k}}\right\}$ such that

$$
\begin{aligned}
& w_{m_{k}} \rightarrow w \text { in } H, \\
& w_{m_{k}} \rightarrow w \text { in } L^{2}(\Omega),
\end{aligned}
$$




$$
w_{m_{k}} \rightarrow w \text { a.e. in } \Omega .
$$

i) Arguing by contradiction, if $w=0$, then $w_{m_{k}} \rightarrow 0$ in $L^{2}(\Omega)$ and

$$
\frac{D J\left(u_{m_{k}}\right)\left(u_{m_{k}}\right)}{\left\|u_{m_{k}}\right\|_{H}} \rightarrow 0 \text { (from definition of PS sequence). }
$$

So, a fortiori

$$
\frac{D J\left(u_{m_{k}}\right)\left(u_{m_{k}}\right)}{\left\|u_{m_{k}}\right\|_{H}^{2}} \rightarrow 0
$$

This yields

$$
\begin{array}{r}
\int_{\Omega} h(x)\left|\nabla w_{m_{k}}\right|^{2} d x-\int_{\Omega} \frac{f\left(x, u_{m_{k}}\right)}{u_{m_{k}}} w_{m_{k}}^{2} d x \rightarrow 0, \\
\left\|w_{m_{k}}\right\|_{H}^{2}-\int_{\Omega} \frac{f\left(x, u_{m_{k}}\right)}{u_{m_{k}}} w_{m_{k}}^{2} d x \rightarrow 0, \\
1-\int_{\Omega} \frac{f\left(x, u_{m_{k}}\right)}{u_{m_{k}}} w_{m_{k}}^{2} d x \rightarrow 0 .
\end{array}
$$

Since $\frac{f\left(x, u_{m_{k}}\right)}{u_{m_{k}}}$ is bounded and $w_{m_{k}} \rightarrow 0$ in $L^{2}(\Omega)$, we get relation $1=0$. Hence we must have $w \neq 0$.

ii) Knowing that $\frac{D J\left(u_{m_{k}}\right)(\varphi)}{\|\varphi\|\left\|u_{m_{k}}\right\|} \rightarrow 0, \forall \varphi \in H, \varphi \neq 0$. We deduce

$$
\frac{\int_{\Omega} h(x) \nabla u_{m_{k}} \cdot \nabla \varphi d x-\int_{\Omega} f\left(x, u_{m_{k}}\right) \varphi d x}{\left\|u_{m_{k}}\right\|} \rightarrow 0, \forall \varphi \in H .
$$

Since $f(x, s)=0$ for $s \leq 0$

$$
\begin{aligned}
\frac{\int_{\Omega} h(x) \nabla u_{m_{k}} \cdot \nabla \varphi d x-\int_{\Omega} f\left(x, u_{m_{k}}^{+}\right) \varphi d x}{\left\|u_{m_{k}}\right\|} & \rightarrow 0, \\
\int_{\Omega} h(x) \nabla w_{m_{k}} \cdot \nabla \varphi d x-\int_{\Omega} \frac{f\left(x, u_{m_{k}}^{+}\right)}{u_{m_{k}}^{+}} \frac{u_{m_{k}}^{+}}{\left\|u_{m_{k}}^{+}\right\|} \varphi d x & \rightarrow 0 .
\end{aligned}
$$

Since $\frac{f\left(x, u_{m_{k}}^{+}\right)}{u_{m_{k}}^{+}}$is bounded, it has a subsequence still denoted by $\frac{f\left(x, u_{m_{k}}^{+}\right)}{u_{m_{k}}^{+}}$, it converges weakly in $L^{2}$ to some function $\theta \in L^{\infty}$. Then,

$$
\begin{gathered}
\int_{\Omega} h(x) \nabla w_{m_{k}} \cdot \nabla \varphi d x-\int_{\Omega} \frac{f\left(x, u_{m_{k}}^{+}\right)}{u_{m_{k}}^{+}} w_{m_{k}}^{+} \varphi d x \rightarrow 0, \\
\int_{\Omega} h(x) \nabla w \cdot \nabla \varphi d x-\int_{\Omega} \theta(x) w^{+} \varphi d x=0, \forall \varphi \in H .
\end{gathered}
$$

Choosing $\varphi=w^{-}$we have

$$
\begin{gathered}
\int_{\Omega} h(x) \nabla w \cdot \nabla w^{-} d x-\int_{\Omega} \theta(x) w^{+} w^{-} d x=0 \\
\int_{\Omega} h(x)\left|\nabla w^{-}\right|^{2} d x=0
\end{gathered}
$$

which implies $w^{-}=0$ then $w \geq 0$. 
So $w \geq 0$ satisfies the equation

$$
-\operatorname{div}(h(x) \nabla w)=\theta(x) w \text { in } \Omega .
$$

Moreover, for any $\Omega^{\prime} \subset \subset \Omega$, we have $h \in L_{l o c}^{1}\left(\Omega^{\prime}\right), w(x) \neq 0, w(x) \geq 0$ in $\Omega^{\prime}$ and

$$
-\operatorname{div}(h(x) \nabla w)=\theta(x) w \text { in } \Omega^{\prime} .
$$

By the Hanark inequality (see [14] Theorem 8.20 and Corollary 8.21), it follows that $w(x)>0$ in $\Omega$. This implies that $w(x)>0$ in $\Omega$.

iii) Since $w>0, u_{m_{k}} \rightarrow+\infty$ a.e. in $\Omega$. So

$$
\begin{gathered}
\frac{f\left(x, u_{m_{k}}\right)}{u_{m_{k}}} \rightarrow \beta(x) \text { a.e. } x \in \Omega, \\
\frac{f\left(x, u_{m_{k}}\right)}{u_{m_{k}}} \rightarrow \theta(x) \text { in } L^{2}(\Omega)
\end{gathered}
$$

this yields $\beta(x)=\theta(x)$. Then $w$ verifies the equation

$$
\int_{\Omega} h(x) \nabla w \nabla \varphi d x=\int_{\Omega} \beta(x) w \varphi d x \text { for all } \varphi \in H
$$

so

$$
-\operatorname{div}(h(x) \nabla w)=\beta(x) w \text { in } \Omega .
$$

The proof of Proposition 3.2 is complete.

Proposition 3.3. Assuming hypotheses F1)-F4) are fulfilled. Then the functional $J: H \rightarrow \mathbb{R}$ is defined by (2.4) satisfies the Palais-Smale condition on $H$.

Proof. Let $\left\{u_{m}\right\}$ be a sequence in $H$ such that

$$
\lim _{m \rightarrow \infty} J\left(u_{m}\right)=c, \quad \lim _{m \rightarrow+\infty}\left\|D J\left(u_{m}\right)\right\|_{H^{*}}=0 .
$$

First, we shall prove that $\left\{u_{m}\right\}$ is bounded in $H$. We suppose by contradiction that $\left\{u_{m}\right\}$ is not bounded in $H$. Then there exists a subsequence still denoted $\left\{u_{m}\right\}$ such that $\left\|u_{m}\right\|_{H} \rightarrow+\infty$ as $m \rightarrow+\infty$. Then putting $w_{m}=\frac{u_{m}}{\left\|u_{m}\right\|_{H}}$, by Proposition 3.2, we have the subsequence $\left\{w_{m_{k}}\right\}$ of $\left\{w_{m}\right\}$ satisfying $w_{m_{k}} \rightarrow w$ in $\mathrm{H}$ and

i) $w \neq 0$ in $\Omega$,

ii) $w>0$ in $\Omega$,

iii) $-\operatorname{div}(h(x) \nabla w)=\beta(x) w$ in $\Omega$.

Hence

So we have

$$
\int_{\Omega} h(x)|\nabla w|^{2} d x=\int_{\Omega} \beta(x) w^{2} d x
$$

$$
1=\frac{\int_{\Omega} h(x)|\nabla w|^{2} d x}{\int_{\Omega} \beta(x) w^{2} d x} .
$$


Recall $\Omega_{\beta}=\{x \in \Omega: \beta(x)>0\} \subset \Omega$, we deduce

$$
\begin{aligned}
1 & =\frac{\int_{\Omega} h(x)|\nabla w|^{2} d x}{\int_{\Omega} \beta(x) w^{2} d x} \geq \frac{\int_{\Omega} h(x)|\nabla w|^{2} d x}{\int_{\Omega_{\beta}} \beta(x) w^{2} d x} \geq \frac{\int_{\Omega_{\beta}} h(x)|\nabla w|^{2} d x}{\int_{\Omega_{\beta}} \beta(x) w^{2} d x} \\
& \geq \inf _{u \in H\left(\Omega_{\beta}\right)} \frac{\int_{\Omega_{\beta}} h(x)|\nabla u|^{2} d x}{\int_{\Omega_{\beta}} \beta(x) u^{2} d x}=\Lambda_{\beta} .
\end{aligned}
$$

By F3) we have a contradiction. So we deduce that all Palais Smale sequences of the functional $J$ are bounded in $H$.

Next we prove that $\left\{u_{m}\right\}$ has a subsequence converging strongly in $H$. Since $\left\{u_{m}\right\}$ is bounded in $H, H$ is a Hilbert space, there exists a subsequence $\left\{u_{m_{k}}\right\}$ such that it converges weakly to some $u$ in $H$ and $\left\{u_{m_{k}}\right\}$ converge strongly in $L^{2}(\Omega)$. Then by Proposition 2.2 we find that

$$
T(u) \leq \lim _{k \rightarrow \infty} \inf T\left(u_{m_{k}}\right) .
$$

Now we prove $\lim _{k \rightarrow+\infty} T\left(u_{m_{k}}\right)=T(u)$. Indeed,

$$
\left\langle D T\left(u_{m_{k}}\right), u_{m_{k}}-u\right\rangle=\left\langle D J\left(u_{m_{k}}\right), u_{m_{k}}-u\right\rangle+\left\langle D P\left(u_{m_{k}}\right), u_{m_{k}}-u\right\rangle .
$$

By the definition of (PS) sequence we have

$$
\lim _{k \rightarrow+\infty}\left\langle D J\left(u_{m_{k}}\right), u_{m_{k}}-u\right\rangle=0 .
$$

From F2)

$$
\begin{aligned}
\left|\left\langle D P\left(u_{m_{k}}\right), u_{m_{k}}-u\right\rangle\right| & =\left|\int_{\Omega} f\left(x, u_{m_{k}}\right)\left(u_{m_{k}}-u\right) d x\right| \\
& \leq \int_{\Omega}\left|\frac{f\left(x, u_{m_{k}}\right)}{u_{m_{k}}}\right|\left|u_{m_{k}}\right|\left|u_{m_{k}}-u\right| d x \\
& \leq C\left(\int_{\Omega}\left|u_{m_{k}}\right|^{2} d x\right)^{\frac{1}{2}} \cdot\left(\int_{\Omega}\left|u_{m_{k}}-u\right|^{2} d x\right)^{\frac{1}{2}} .
\end{aligned}
$$

Since $u_{m_{k}} \rightarrow u$ in $L^{2}(\Omega)$ we have

$$
\lim _{k \rightarrow+\infty}\left\langle D P\left(u_{m_{k}}\right), u_{m_{k}}-u\right\rangle=0 .
$$

Hence

$$
\lim _{k \rightarrow+\infty}\left\langle D T\left(u_{m_{k}}\right), u_{m_{k}}-u\right\rangle=0 .
$$

On the other hand, since $T$ is convex the following inequality holds true

$$
T(u)-T\left(u_{m_{k}}\right) \geq\left\langle D T\left(u_{m_{k}}\right), u-u_{m_{k}}\right\rangle .
$$

Letting $k \rightarrow+\infty$ we have

$$
\begin{aligned}
T(u)-\lim _{k \rightarrow+\infty} T\left(u_{m_{k}}\right) & =\lim _{k \rightarrow+\infty}\left[T(u)-T\left(u_{m_{k}}\right)\right] \\
& \geq \lim _{k \rightarrow+\infty}\left\langle D T\left(u_{m_{k}}\right), u-u_{m_{k}}\right\rangle=0 .
\end{aligned}
$$


This implies that

$$
T(u) \geq \lim _{k \rightarrow+\infty} T\left(u_{m_{k}}\right) .
$$

From (3.9), (3.10) we get $\lim _{k \rightarrow+\infty} T\left(u_{m_{k}}\right)=T(u)$.

Now we prove that the sequence $\left\{u_{m_{k}}\right\}$ converges strongly to $u$ in $H$. Indeed, we suppose by contradiction that $\left\{u_{m_{k}}\right\}$ is not converges strongly to $u$ in $H$. Then there exist a constant $\epsilon_{0}>0$ and a subsequence $\left\{u_{m_{k_{j}}}\right\}$ of $\left\{u_{m_{k}}\right\}$ such that $\left\|u_{m_{k_{j}}}-u\right\|_{H} \geq \epsilon_{0}$ for $j=1,2, \ldots$

Recalling inequality

$$
\left|\frac{\alpha+\beta}{2}\right|^{2}+\left|\frac{\alpha-\beta}{2}\right|^{2}=\frac{1}{2}\left(|\alpha|^{2}+|\beta|^{2}\right), \quad \forall \alpha, \beta \in \mathbb{R} .
$$

We deduce that for any $j=1,2, \ldots$

$$
\begin{aligned}
& \frac{1}{2} T\left(u_{m_{k_{j}}}\right)+\frac{1}{2} T(u)-T\left(\frac{u_{m_{k_{j}}}+u}{2}\right) \\
\geq & \frac{1}{4}\left\|u_{m_{k_{j}}}-u\right\|_{H}^{2}=\left(\frac{\epsilon_{0}}{2}\right)^{2} .
\end{aligned}
$$

Again instead of the remark that since $\left\{\frac{u_{m_{k_{j}}}+u}{2}\right\}$ converges weakly to $u$ in $H$, applying Proposition 2.2 we have

$$
T(u) \leq \lim _{j \rightarrow+\infty} \inf T\left(\frac{u_{m_{k_{j}}}+u}{2}\right) .
$$

Then from (3.11), letting $j \rightarrow \infty$ we obtain

$$
T(u)-\lim _{j \rightarrow+\infty} \inf T\left(\frac{u_{m_{k_{j}}}+u}{2}\right) \geq\left(\frac{\epsilon_{0}}{2}\right)^{2}>0
$$

which is a contradiction. Therefore, $\left\{u_{m_{k}}\right\}$ converges strongly to $u$ in $H$. Thus the functional $J$ satisfies the Palais-Smale condition on $H$. The proof of Proposition 3.3 is complete.

Proposition 3.4. i) $J(0)=0$.

ii) The acceptable set $G=\left\{\gamma \in C([0,1], H): \gamma(0)=0, \gamma(1)=u_{0}\right\}$ is not empty (with $u_{0}$ in Proposition 3.1).

Proof. i) Follows from F1) and the definition of $J$ we have $\mathrm{J}(0)=0$.

ii) Let $\gamma(t)=t u_{0}$, so $\gamma(0)=0, \gamma(1)=u_{0}$, then $\gamma(t) \in G$ and $G \neq \emptyset$.

Proof of Theorem 3.1. By Propositions 3.1-3.4, all assumptions of the variations of the mountain pass theorem introduced in [12] are satisfied. Therefore there exists $\tilde{\mathrm{w}} \in H$ such that

$$
0<\alpha \leq J(\tilde{\mathrm{w}})=\inf \{\max J(\gamma([0,1])): \gamma \in G\}
$$


and $\langle D J(\tilde{\mathrm{w}}), v\rangle=0$ for all $v \in H$, i.e., $\tilde{\mathrm{w}}$ is a weak solution of the problem (1.1). Moreover since $J(\tilde{\mathrm{w}})>0=J(0), \tilde{\mathrm{w}}$ is a nontrivial weak solution of the problem (1.1). We have

$$
\int_{\Omega} h(x) \nabla \tilde{\mathrm{w}} \nabla \varphi d x-\int_{\Omega} f(x, \tilde{\mathrm{w}}) \varphi d x=0, \quad \forall \varphi \in H .
$$

Choose $\varphi=\tilde{\mathrm{w}}^{-}, f(x, w)=0$ as $w \leq 0$. So we obtain

$$
\int_{\Omega} h(x) \nabla \tilde{\mathrm{w}} \nabla \tilde{\mathrm{w}}^{-} d x=0 \text { or }\left\|\tilde{\mathrm{w}}^{-}\right\|_{H}=0 .
$$

Then $\tilde{\mathrm{w}} \geq 0$, is a weak solution non-negative non-trivial of the problem (1.1). Theorem 3.1 is completely proved.

\section{References}

[1] M. Alif and P. Omari, On a p-Laplace Neumann problem with asymptotically asymmetric perturbations, Nonlinear Anal. 5151 (2002), no. 2, 369-389.

[2] H. Amann and T. Laetsch, Positive solutions of convex nonlinear eigenvalue problems, Indiana Univ. Math. J. 25 (1976), no. 3, 259-270.

[3] A. Ambrosetti and P. Hess, Positive solutions of asymptotically linear elliptic eigenvalue problems, J. Math. Anal. Appl. 73 (1980), no. 2, 411-422.

[4] G. Anello, Existence of infinitely many weak solutions for a Neumann problem, Nonlinear Anal. 57 (2004), no. 2, 199-209.

[5] H. Berestycki, I. Capuzzo Dolcetta, and L. Nirenberg, Variational methods for indefinite superlinear homogeneous elliptic problems, NoDEA 2 (1995), no. 4, 553-572.

[6] _ Superlinear indefinite elliptic problems and nonlinear Liouville theorems, Topol. Methods Nonlinear Anal. 4 (1994), no. 1, 59-78.

[7] K. J. Brown and A. Tertikas, On the bifurcation of radially symmetric steady-state solutions arising in population genetics, SIAM J. Math. Anal 22 (1991), no. 2, 400-413.

[8] K. C. Chang, Solutions of asymptotically linear operator equations via Morse theory, Comm. Pure Appl. Math. 34 (1981), no. 5, 693-712.

[9] N. T. Chung and H. Q. Toan, Existence result for nonuniformly degenerate semilinear elliptic systems in $R^{N}$, Glassgow. Math. J. 51 (2009), 561-570.

[10] D. G. Costa and C. A. Magalhães, Variational elliptic problems which are nonquadratic at infinity, Nonlinear Anal. 23 (1994), no. 11, 1401-1412.

[11] T.-L. Dinu, Subcritical perturbations of resonant linear problem with sign-changing potential, Electron. J. Differential Equations 2005 (2005), no. 117, 15 pp.

[12] D. M. Duc, Nonlinear singular elliptic equation, J. London Math. Soc. (2) 40 (1989), no. 3, 420-440.

[13] W. H. Fleming, A selection-Migration model in population genetics, J. Math. Biol. 2 (1975), no. 3, 219-233.

[14] D. Gilbarg and N. Trudinger, Elliptic Partial Differential Equations of Second Order, Springer Verlag, Berlin, 1998.

[15] P. Hess, Multiple solutions of asymptotically linear elliptic boundary value problems, Proc. Equadiff IV, Prague 1977, Lecture notes in Math. 703, Springer Verlag, New York, 1979.

[16] P. Hess and T. Kato, On some linear and nonlinear eigenvalue problems with an indefinite weight function, Comm. Partial Differential Equations 5 (1980), no. 10, 999-1030.

[17] M. Lucia, P. Magrone, and H.-S. Zhou, A Dirichlet problem with asymptotically linear and changing sign nonlinearity, Rev. Mat. Complut. 16 (2003), no. 2, 465-481. 
[18] B. Ricceri, Infinity many solutions of the Neumann problem for elliptic equations involving the p-Laplacian, Bull. London Math. Soc. 33 (2001), 331-340.

[19] C. L. Tang, Solvability of Neumann problem for elliptic equations at resonnance, Nonlinear Anal. 44 (2001), 325-335.

[20] _ Some existence theorems for the sublinear Neumann boundary value problem, Nonlinear Anal. 48 (2002), no. 7, 1003-1011.

[21] H. Q. Toan and N. Q. Anh, Multiplicity of weak solutions for a class of nonuniformly nonlinear elliptic equation of p-Laplacian type, Nonlinear Anal. 70 (2009), 1536-1546.

[22] H. Q. Toan and N. T. Chung, Existence of weak solutions for a class of nonuniformly nonlinear elliptic equations in unbounded domains, Nonlinear Anal. 70 (2009), no. 11, 3987-3996.

[23] H. S. Zhou, Positive solutions for a semilinear elliptic equation which is almost linear at infinity, Z. Angew. Math. Phys. 49 (1998), no. 6, 896-906.

[24] - An application of a mountain pass theorem, Acta Math. Sinica 18 (2002), no. $1,27-36$.

Trinh Thi Minh HANG

DEPARTMENT OF INFORMATICS

Hanoi University of Civil Engineering

55 Giai Phong, Hanoi, Vietnam

E-mail address: quoctrung032007@yahoo.com

HoAng QuOC TOAN

Department of Mathematics

Hanoi University of SCIENCE

334 Nguyen Trai, Hanoi, Vietnam

E-mail address: hq_toan@yahoo.com 